\title{
Range of outputs precise of digits rounding in SPSS and MS Excel
}

\author{
Zaher Saif Mohammed Al-Hashami \\ CS Instructor-Muscat, Sultanate of Oman \\ E-mail: acade.re.z@gmail.com
}

Copyright (c) 2014 Zaher Saif Mohammed Al-Hashami. This is an open access article distributed under the Creative Commons Attribution License, which permits unrestricted use, distribution, and reproduction in any medium, provided the original work is properly cited.

\begin{abstract}
The statistical operations done by many specialist programs, by them can do these operations fluently and precisely. There are many functions in such programs can calculate in SAS, STAT and the analytical program SPSS. There is Microsoft Excel program that is calculate like these functions. The level of some programs may be different than others within these functions they are calculating. From these functions are Sum, Average, Maximum and Minimum. Round function is also from these functions that can measure it's accuracy through this research. In this research i chose ten digits numbers and I also chose three criteria under, equal and more than 5. According to that the rounding operations are done based on if wanted decimal place is less, equal or higher than 5. Rounding applied on ten digits using SPSS and MS Excel programs. The outputs findings are the same except that Microsoft Excel is truncating the last zeroes of the digit after the decimal point. Wherever the decimal place specified in the digit is want it will truncate after the decimal point. The SPSS is more precise than MS Excel based on the decimal place in the digit number wanted statistically and analytically.
\end{abstract}

Keywords: Decimal Place, Digit, Excel, Function, Round.

\section{Introduction}

Functions in many statistical programs like MS Excel and SPSS programs are count many kinds of numbers like integers, natural, real and digits. The results of such as these functions are supposed to be same outputs in MS Excel and in SPSS programs. The digits numbers rounding are also supposed to be the same in many statistic programs like SPSS, SAS, STAT and MS Excel.

Ted French said in Spreadsheet.about.com: rounding a number means altering it to a specific size. Rounding integers (whole numbers) involves rounding them up or down to the nearest multiple of 10, while rounding decimals reduces them to a set number of decimal places [1].

According to Poes and Matthew Joseph,2011 [2] they used SPSS program to export some values to MS excel, by save it as Excel file, when they do this, it's truncating the values down to only one digit after the decimal place. It also is not rounding correctly, as some values which should be appropriately rounded up, are being rounded down. They said as example, a value of 6.547 becomes 6.5 instead of 6.6.

The digits numbers in both SPSS and MS Excel are supposed to round up into the same truncate numbers, whatever decimal place must convert.

In SPSS the decimals are valid for numeric variables only. It specifies the number of decimals to be kept for a variable. All the extra decimals will be rounded up and the rounded numbers will be used in all the analysis, so should be careful to specify the number of decimals to fit in the precision the researcher want.

Professor Matthew in his study name: Reporting Statistics in APA Style [3] using SPSS, about using rounding appropriately said round number to one or two decimal place he said, must keep in mind that fewer decimal places are easier to comprehend. He said must consider rescaling measurements required for more two decimal places.

A scientific fact stipulates that, when rounding numbers especially digits numbers, these numbers should be rounded up as far as rounded down. The rounding precisely must examine the first digit to be truncated. If the digits are $6,7,8$, or 9 , round the number will be up. When the digits are 1,2,3, or 4 the rounding will be down. Then if this digit is a 5, you should look to the remaining digits beyond the 5 to see if they are all zeroes. 
If they are not all zeroes, then the number does not end in an exact 5 and should be rounded up. If all remaining digits to the right are zero or in other word there are no additional digits available to the right of the 5, then the number in its current precision is an exact 5. Then in this state, that the number should be rounded up as often as it is rounded down. This is a research done by choosing ten samples digits if they are less, equal and more 5 in the decimal place to check the round result through these three cases in SPSS and MS Excel.

\section{Method}

The concept of this research is calculates rounding and truncate in SPSS and round function in MS Excel for 10 random digits numbers and comparing the results in the two programs with the same digits numbers. Compare the rounding outputs in both programs in four decimal places. The research Methodology is by choosing 10 random digits numbers with four decimal places and do the rounding less than 5, equal 5 (after decimal place numbers) and bigger than 5 . These rounding could do it in SPSS and MS Excel with the same ten random digits numbers in both. Then could compare the rounding and truncate results of the same digits numbers in the two programs.

The rounding is done according to 5 number position whether it in under, equal (after decimal place number to get rounding in the decimal place number) and higher than 5, if the number after the decimal place is bigger or less than 5. In the below tables can see the decimal place in bold color. In case of that it is equal 5; the position of the five numbers must be after the decimal place.

In MS Excel the rounding is done by ROUND function by inter the digit and then inter the decimal place. In SPSS must consider how many decimal places should inter when interring the variables.

\section{SPSS rounding}

\subsection{Under 5}

Table 1.3.1: The under 5 digits number and their decimal places

\begin{tabular}{|c|c|c|c|c|c|c|c|c|c|c|c|}
\hline Digits Numbers & & 1 & 2 & 3 & 4 & 5 & 6 & 7 & 8 & 9 & 10 \\
\hline \multirow{4}{*}{ 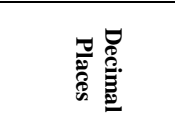 } & 1 & 26.42 & 77.32 & 12.22 & 49.14 & 66.01 & 33.34 & 70.44 & 41.13 & 75.03 & 91.11 \\
\hline & 2 & 76.343 & 86.444 & 90.213 & 24.433 & 55.420 & 78.222 & 40.302 & 11.101 & 20.410 & 30.321 \\
\hline & 3 & 67.3421 & 74.3214 & 46.2313 & 90.1020 & 45.2333 & 76.2003 & 31.2304 & 87.2113 & 50.4444 & 10.3423 \\
\hline & 4 & 24.10201 & 76.34321 & 11.34441 & 90.20302 & 43.12320 & 21.40400 & 87.22323 & 21.34000 & 10.20111 & 99.23421 \\
\hline
\end{tabular}

The SPSS outputs rounding if the decimal places numbers are less than 5 in the four decimal places like in table 2.3.1:

Table 2.3.1: SPSS under 5 digits number rounding results

\begin{tabular}{|c|c|c|c|c|c|c|c|c|c|}
\hline \multicolumn{10}{|c|}{ The first decimal place rounding result } \\
\hline one & two & three & four & five & six & seven & eight & nine & ten \\
\hline 26.4 & 77.3 & 12.2 & 49.1 & 66.0 & 33.3 & 70.4 & 41.1 & 75.0 & 91.1 \\
\hline \multirow{2}{*}{\multicolumn{10}{|c|}{ The second decimal place rounding result }} \\
\hline & & & & & & & & & \\
\hline one & two & three & four & five & six & seven & eight & nine & ten \\
\hline 76.34 & 86.44 & 90.21 & 24.43 & 55.42 & 78.22 & 40.30 & 11.10 & 20.41 & 30.32 \\
\hline \multicolumn{10}{|c|}{ The third decimal place rounding result } \\
\hline one & two & three & four & five & six & seven & eight & nine & ten \\
\hline 67.342 & 74.321 & 46.231 & 90.102 & 45.233 & 76.200 & 31.230 & 87.211 & 50.444 & 10.342 \\
\hline \multicolumn{10}{|c|}{ The fourth decimal place rounding result } \\
\hline one & two & three & four & five & six & seven & eight & nine & ten \\
\hline 24.1020 & 76.3432 & 11.3444 & 90.2030 & 43.1232 & 21.4040 & 87.2232 & 21.3400 & 10.2011 & 99.2342 \\
\hline
\end{tabular}

\subsection{Equal 5 after decimal place number}

In this case the five numbers must be after the number of decimal place to get the rounding outputs:

Table 1.3.2: The Equal 5 digits and their decimal places

\begin{tabular}{|c|c|c|c|c|c|c|c|c|c|c|c|}
\hline Digits Numbers & & 1 & 2 & 3 & 4 & 5 & 6 & 7 & 8 & 9 & 10 \\
\hline \multirow{4}{*}{ 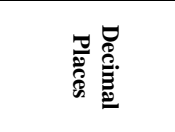 } & 1 & 26.25 & 77.35 & 12.05 & 49.75 & 66.85 & 33.75 & 70.85 & 41.95 & 75.15 & 91.45 \\
\hline & 2 & 76.345 & 86.465 & 90.235 & 24.495 & 55.405 & 78.295 & 40.305 & 11.115 & 20.555 & 30.595 \\
\hline & 3 & 67.3485 & 74.3205 & 46.2365 & 90.1045 & 45.2355 & 76.2035 & 31.2395 & 87.2175 & 50.4495 & 10.3435 \\
\hline & 4 & 24.10215 & 76.34315 & 11.34405 & 90.20355 & 43.12375 & 21.40445 & 87.22385 & 21.34025 & 10.20165 & 99.23445 \\
\hline
\end{tabular}


In the table 2.3.2 the SPSS outputs rounding if the decimal places are equal 5 and put it after decimal place number in the four decimal places are:

Table 2.3.2: SPSS equal 5 digits number rounding results

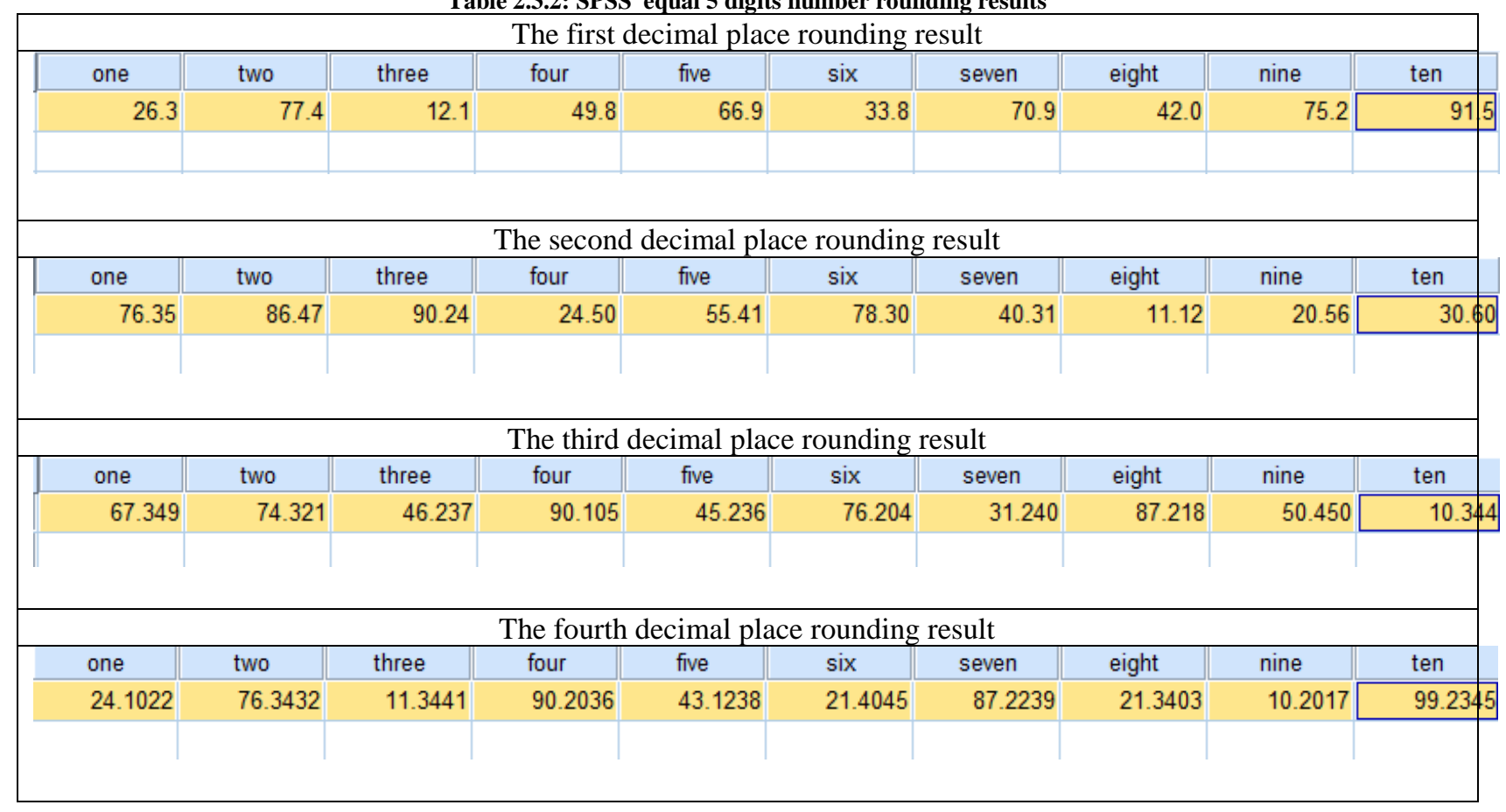

\subsection{Higher than 5}

Table 1.3.3: The higher than 5 digits number and their decimal places

\begin{tabular}{|c|c|c|c|c|c|c|c|c|c|c|c|}
\hline Digits Numbers & & $\mathbf{1}$ & 2 & 3 & 4 & 5 & 6 & 7 & 8 & 9 & 10 \\
\hline \multirow{4}{*}{ 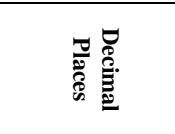 } & 1 & 26.67 & 77.76 & 12.99 & 49.87 & 66.98 & 33.77 & 70.88 & 41.69 & 75.97 & 91.86 \\
\hline & 2 & 76.377 & 86.486 & 90.298 & 24.468 & 55.476 & 78.269 & 40.387 & 11.166 & 20.596 & 30.589 \\
\hline & 3 & 67.3488 & 74.3279 & 46.2366 & 90.1097 & 45.2369 & 76.2076 & 31.2369 & 87.2179 & 50.4489 & 10.3496 \\
\hline & 4 & 24.10268 & 76.34377 & 11.34496 & 90.20376 & 43.12397 & 21.40469 & 87.22378 & 21.34069 & 10.20186 & 99.23497 \\
\hline
\end{tabular}

The SPSS outputs rounding if the decimal places numbers are higher than 5 in the four decimal places are in this table:

Table 2.3.3: SPSS Higher than 5 digits rounding results

\begin{tabular}{|c|c|c|c|c|c|c|c|c|c|}
\hline \multicolumn{10}{|c|}{ The first decimal place rounding result } \\
\hline one & two & three & four & five & six & seven & eight & nine & ten \\
\hline 26.7 & 77.8 & 13.0 & 49.9 & 67.0 & 33.8 & 70.9 & 41.7 & 76.0 & 91.9 \\
\hline \multicolumn{10}{|c|}{ The second decimal place rounding result } \\
\hline one & two & three & four & five & six & seven & eight & nine & ten \\
\hline 76.38 & 86.49 & 90.30 & 24.47 & 55.48 & 78.27 & 40.39 & 11.17 & 20.60 & 30.59 \\
\hline \multicolumn{10}{|c|}{ The third decimal place rounding result } \\
\hline one & two & three & four & five & six & seven & eight & nine & ten \\
\hline 67.349 & 74.328 & 46.237 & 90.110 & 45.237 & 76.208 & 31.237 & 87.218 & 50.449 & 10.350 \\
\hline \multicolumn{10}{|c|}{ The fourth decimal place rounding result } \\
\hline one & two & three & four & five & six & seven & eight & nine & ten \\
\hline 24.1027 & 76.3438 & 11.3450 & 90.2038 & 43.1240 & 21.4047 & 87.2238 & 21.3407 & 10.2019 & 99.2350 \\
\hline & & & & & & & & & \\
\hline
\end{tabular}

\section{MS excel rounding}

\subsection{Under 5}


Table 1.4.1: Under 5 digits and their decimal places

\begin{tabular}{|c|c|c|c|c|c|c|c|c|c|c|c|}
\hline Digits Numbers & & 1 & 2 & 3 & 4 & 5 & 6 & 7 & 8 & 9 & 10 \\
\hline \multirow{4}{*}{ 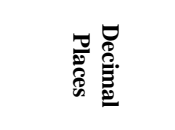 } & 1 & 26.42 & 77.32 & 12.22 & 49.14 & 66.01 & 33.34 & 70.44 & 41.13 & 75.03 & 91.11 \\
\hline & 2 & 76.343 & 86.444 & 90.213 & 24.433 & 55.420 & 78.222 & 40.302 & 11.101 & 20.410 & 30.321 \\
\hline & 3 & 67.3421 & 74.3214 & 46.2313 & 90.1020 & 45.2333 & 76.2003 & 31.2304 & 87.2113 & 50.4444 & 10.3423 \\
\hline & 4 & 24.10201 & 76.34321 & 11.34441 & 90.20302 & 43.12320 & 21.40400 & 87.22323 & 21.34000 & 10.20111 & 99.23421 \\
\hline
\end{tabular}

The same method rounding in SPSS, in MS Excel outputs rounding after consideration that the decimal places are less than 5 in the four decimal places are:

Table 2.4.1: Excel under 5 digits rounding results

\begin{tabular}{|c|c|c|c|c|c|c|c|c|c|}
\hline \multicolumn{10}{|c|}{ The first decimal place rounding result } \\
\hline 1 & 2 & 3 & 4 & 5 & 6 & 7 & 8 & 9 & 10 \\
\hline 91.1 & 75 & 41.1 & 70.4 & 33.3 & 66 & 49.1 & 12.2 & $\overline{77.3}$ & 26.4 \\
\hline \multicolumn{10}{|c|}{ The second decimal place rounding result } \\
\hline 1 & 2 & 3 & 4 & 5 & 6 & 7 & 8 & 9 & 10 \\
\hline 30.32 & 20.41 & 11.1 & 40.3 & 78.22 & 55.42 & 24.43 & 90.21 & 86.44 & 76.34 \\
\hline \multicolumn{10}{|c|}{ The third decimal place rounding result } \\
\hline 1 & 2 & 3 & 4 & 5 & 6 & 7 & 8 & 9 & 10 \\
\hline 10.342 & 50.444 & 87.211 & 31.23 & 76.2 & 45.233 & 90.102 & 46.231 & 74.321 & 67.342 \\
\hline \multicolumn{10}{|c|}{ The fourth decimal place rounding result } \\
\hline 1 & 2 & 3 & 4 & 5 & 6 & 7 & 8 & 9 & 10 \\
\hline 99.2342 & 10.2011 & 21.34 & 87.2232 & 21.404 & 43.1232 & 90.203 & 11.3444 & 76.3432 & 24.102 \\
\hline
\end{tabular}

\subsection{Equal 5 after decimal place number}

The same in SPSS, this rounding in MS Excel is consider 5 number after the decimal place number

Table 1.4.2: Equal 5 digits and their decimal places

\begin{tabular}{|c|c|c|c|c|c|c|c|c|c|c|c|}
\hline Digits Numbers & & 1 & 2 & 3 & 4 & 5 & 6 & 7 & 8 & 9 & 10 \\
\hline \multirow{4}{*}{ 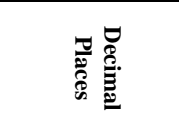 } & 1 & 26.25 & 77.35 & 12.05 & 49.75 & 66.85 & 33.75 & 70.85 & 41.95 & 75.15 & 91.45 \\
\hline & 2 & 76.345 & 86.465 & 90.235 & 24.495 & 55.405 & 78.295 & 40.305 & 11.115 & 20.555 & 30.595 \\
\hline & 3 & 67.3485 & 74.3205 & 46.2365 & 90.1045 & 45.2355 & 76.2035 & 31.2395 & 87.2175 & 50.4495 & 10.3435 \\
\hline & 4 & 24.10215 & 76.34315 & 11.34405 & 90.20355 & 43.12375 & 21.40445 & 87.22385 & 21.34025 & 10.20165 & 99.23445 \\
\hline
\end{tabular}

In MS Excel outputs rounding also can consider if the decimal places are equal 5 and put it after decimal place number in the four decimal places are the below table:

Table 2.4.2: Excel equal 5 digits rounding results

\begin{tabular}{|c|c|c|c|c|c|c|c|c|c|}
\hline \multicolumn{10}{|c|}{ The first decimal place rounding result } \\
\hline 1 & 2 & 3 & 4 & 5 & 6 & 7 & 8 & 9 & 10 \\
\hline 91.5 & 75.2 & 42 & 70.9 & 33.8 & 66.9 & 49.8 & 12.1 & 77.4 & 26.3 \\
\hline & & & & & & & & & \\
\hline \multicolumn{10}{|c|}{ The second decimal place rounding result } \\
\hline 1 & 2 & 3 & 4 & 5 & 6 & 7 & 8 & 9 & 10 \\
\hline 30.6 & 20.56 & 11.12 & 40.31 & 78.3 & 55.41 & 24.5 & 90.24 & 86.47 & 76.35 \\
\hline \multicolumn{10}{|c|}{ The third decimal place rounding result } \\
\hline 1 & 2 & 3 & 4 & 5 & 6 & 7 & 8 & 9 & 10 \\
\hline 10.344 & 50.45 & 87.218 & 31.24 & 76.204 & 45.236 & 90.105 & 46.237 & 74.321 & 67.349 \\
\hline \multicolumn{10}{|c|}{ The fourth decimal place rounding result } \\
\hline 1 & 2 & 3 & 4 & 5 & 6 & 7 & 8 & 9 & 10 \\
\hline 99.2345 & 10.2017 & 21.3403 & 87.2239 & 21.4045 & 43.1238 & 90.2036 & 11.3441 & 76.3432 & 24.1022 \\
\hline
\end{tabular}




\subsection{Higher than 5}

Table 1.4.3: Higher than 5 digits and their decimal places

\begin{tabular}{|c|c|c|c|c|c|c|c|c|c|c|c|}
\hline Digits Numbers & & 1 & 2 & 3 & 4 & 5 & 6 & 7 & 8 & 0 & 10 \\
\hline \multirow{4}{*}{ 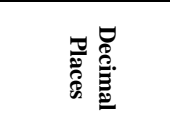 } & $\mathbf{1}$ & 26.67 & 77.76 & 12.99 & 49.87 & 66.98 & 33.77 & 70.88 & 4169 & 7597 & 9186 \\
\hline & 2 & 76.377 & 86.486 & 90.298 & 24.468 & 55.476 & 78.269 & 40.387 & 11.166 & 20.596 & 30.589 \\
\hline & 3 & 67.3488 & 74.3279 & 46.2366 & 90.1097 & 45.2369 & 76.2076 & 31.2369 & 87.2179 & 50.4489 & 10.3496 \\
\hline & 4 & 24.10268 & 76.34377 & 11.34496 & 90.20376 & 43.12397 & 21.40469 & 87.22378 & 21.34069 & 10.20186 & 99.23497 \\
\hline
\end{tabular}

MS Excel outputs rounding if the decimal places numbers are higher than 5 in the four decimal places are in the table 2.4.3:

Table 2.4.3: Excel higher than 5 rounding results

\begin{tabular}{|rrrrrrrrrr|}
\hline \multicolumn{10}{|c|}{ The first decimal place rounding result } \\
\hline $\mathbf{1}$ & $\mathbf{2}$ & $\mathbf{3}$ & $\mathbf{4}$ & $\mathbf{5}$ & $\mathbf{6}$ & $\mathbf{7}$ & $\mathbf{8}$ & $\mathbf{9}$ & $\mathbf{1 0}$ \\
\hline 91.9 & 76 & 41.7 & 70.9 & 33.8 & 67 & 49.9 & 13 & 77.8 & 26.7 \\
\hline $\mathbf{8}$ & \multicolumn{10}{|c|}{ The second decimal place rounding result } \\
\hline $\mathbf{1}$ & $\mathbf{2}$ & $\mathbf{3}$ & $\mathbf{4}$ & $\mathbf{5}$ & $\mathbf{6}$ & $\mathbf{7}$ & $\mathbf{8}$ & $\mathbf{9}$ & $\mathbf{1 0}$ \\
\hline 30.59 & 20.6 & 11.17 & 40.39 & 78.27 & 55.48 & 24.47 & 90.3 & 86.49 & 76.38 \\
\hline $\mathbf{1}$ & $\mathbf{2}$ & $\mathbf{3}$ & $\mathbf{3}$ & $\mathbf{4}$ & $\mathbf{6}$ & $\mathbf{7}$ & $\mathbf{8}$ & $\mathbf{9}$ & $\mathbf{1 0}$ \\
\hline 10.35 & 50.449 & 87.218 & 31.237 & 76.208 & 45.237 & 90.11 & 46.237 & 74.328 & 67.349 \\
\hline $\mathbf{1 0}$ & The fourth decimal place rounding result & & & \\
\hline $\mathbf{1}$ & $\mathbf{2}$ & $\mathbf{3}$ & $\mathbf{4}$ & $\mathbf{5}$ & $\mathbf{6}$ & $\mathbf{7}$ & $\mathbf{8}$ & $\mathbf{9}$ & $\mathbf{1 0}$ \\
\hline 99.235 & 10.2019 & 21.3407 & 87.2238 & 21.4047 & 43.124 & 90.2038 & 11.345 & 76.3438 & 24.1027 \\
\hline
\end{tabular}

\section{Discussion}

The outputs of the rounding less, equal and after decimal place and higher than 5 are all the same results in SPSS and MS Excel, except that MS Excel truncate the zeros by default after the decimal point number if there zeroes after it and in the last one or two or even three numbers.

From the tabulation results can be seen that in the first output of the rounding under 5, which is less than 5 in the second decimal point, can be found the digit 11.10 in SPSS is truncated in MS Excel to 11.1 which is rounding to two decimal places. Also less than 5 outputs can see the digit: 76.200 in SPSS rounded to three decimal places while in MS Excel rounded to 76.2. Another example indicates that MS Excel truncate the digit number if there are zeroes after the decimal point, if there aren't any number only (0000), like 21.3400 when rounded to four decimal places rounded to 21.34 in Microsoft Excel program where in the SPSS statistical program is 21.3400.

In other outputs from the tabulation outputs indicate that MS Excel program is rounding the digits without zeroes after decimal point numbers in the equal and after decimal place is 30.60 in the second decimal place rounding. This digit rounded to 30.6 in Microsoft Excel and in SPSS result got the same digit (30.60). Also 13.240 in the third decimal place rounding can be seen that it is rounded to 13.24 in MS Excel. It could be seen from the first time to the reader that it is rounded to second decimal place, but MS Excel rounding it as third decimal place. 67.0 is a digit rounded to the first decimal place. In SPSS is the same 67.0, in contrast in MS Excel it is rounded to 67. In this rounding for the reader it is an integer while it is rounded to first decimal place.

SPSS is more precise than MS Excel in the last results calculation in considering the digits numbers after the decimal point.

Last instances when rounding to fourth decimal place in this digit: 11.2450 , it is rounded to four decimal places while in MS excel rounded to 11.245 with truncate of the zero after 5 .

When doing a function in SPSS and MS Excel, example for that is Sum function for digits numbers: 56.60000 +34.70000 will equal 91.30000 .

In SPSS statistic program will equal 91.30000. In contrast in MS Excel it equal 91.3 which is rounded to that digit in MS Excel. This function result is rounded without zeroes after decimal point and after first number 3.

Average function can be calculated for $4.3000,4.4000$ and 4.5000 the output of SPSS is 4.4000 and the result in MS Excel is 4.4.

In such Statistical programs, the SPSS program is more precise than MS Excel especially in counting the decimal places after the decimal point. 
This digit: 2.3000000 is indicates how many precise after decimal point is want, example for that, if want two decimal points the result will be 2.30 while the in the MS Excel in 2.3. If wanted is four decimal places the result will be 2.3000 and also in the MS Excel the result is 2.3.

In MS Excel there is no significant zeros are displayed correctly when rounding a number to a certain number of significant digits.

Ted French, 2014 [4] in his article entitled: Excel Round Function, he said that Excel normally rounding the number to the right of the rounding digit determines whether the rounding digit will be rounded up or down.

Ted French also said that MS Excel follows some rules like SPSS program rules when doing the rounding: the first is if the value of the number to the right of the rounding digit is less than five, the rounding digit is left unchanged. The second is if the value of the number to the right of the rounding digit is five or higher, the rounding digit is raised by one.

According to Tim Birkett on Online Excel Tutorial, 2006 [5]: in MS Excel, Round function takes two arguments first the number to be rounded while the second is the number of decimal places to round to. He said also to prevent the format to do not displaying three zeros based on this research outputs, it is suggested to put comma like this: \#,\#\#,;

\section{Conclusion}

From the statistic programs are SPSS, SAS and STAT as well as Microsoft Excel programs that can by it done many statistical operations and many functions. These functions are common between such as these statistical programs and the same rules are followed. Round function in SPSS and MS Excel is shared.

The ten samples digits numbers which are less than 5, more than 5 and equal 5, are all the same outputs got when applying rounding in both SPSS and MS Excel. The same round is gotten with the same results. A SPSS statistical program is more precise than MS Excel when it truncates any zero after decimal point in the digit number. Wherever decimal place is wanted after specific decimal point in the digit will be truncated in Microsoft Excel. Example for that in this research is: 21.34000 rounded to 21.34 , while it wanted the fourth decimal place to round.

In case of calculate some other functions like sum and average also the same results will get after done the function with truncate the zeroes after decimal point whatever number of zeros are there. Based on SPSS that it is more precise and more support in précised results in the calculations. According to what the researcher wants that could assign the decimal place to round the digit number statistically within the range of precision that the researcher wants.

\section{Acknowledgment}

Thank to everyone help me and was helpful to finish this research. I hope that this research is very useful for who looking something about applying functions in SPSS and MS Excel and to go through such as like this scientific researching in IT. I hope to do more researches in IT and CS scope in the future.

\section{References}

[1] French Ted, Rounding Numbers in Excel, the Excel 2007 ROUND function, $2014 . \quad$ Website: spreadsheets.about.com/od/excel2007/qt/excel2007_round.htm

[2] Poes, Joseph Matthew, Truncation of values after Decimal when exporting to Excel, 2011. Website: spssxdiscussion.1045642.n5.nabble.com/Truncation-of-values-after-Decimal-when-exporting-to-Excel-td5068824.html

[3] Matthew Hesson, Reporting statistics in APA Style, A Short guide to handling numbers and statistics in APA Style, 2014. Website: my.ilstu.edu/ mshesso/apa_stats.htm

[4] French Ted, Excel Round Function, rounding numbers in Excel, $2014 . \quad$ Website: spreadsheets.about.com/od/excelfunctions/qt/070809_round.htm

[5] Tim Birkett, Rounding, Online Excel Tutorial, 2006. accountingweb.co.uk/topic/excel-faqs-rounding/493946 